\title{
MedLink: A Mobile Intervention to Address Failure Points in the Treatment of Depression in General Medicine
}

\author{
David C. Mohr, Ph.D. ", Enid Montague, Ph.D. ', Colleen Stiles-Shields ${ }^{*}$, Susan M. Kaiser ${ }^{*}$, \\ Christopher Brenner ${ }^{*}$, Eric Carty-Fickes* , Hannah Palac ${ }^{*}$, Jenna Duffecy ${ }^{*}$ \\ *Center for Behavioral Intervention Technologies, \\ Northwestern University \\ Chicago, Illinois, USA \\ Email: d-mohr@northwestern.edu \\ ${ }^{\dagger}$ Division of General Internal Medicine \\ Northwestern University \\ Chicago, Illinois, USA \\ *Department of Psychiatry \\ University of Illinois \\ Chicago, Illinois, USA
}

\begin{abstract}
Major depression is common, and imposes a high burden in terms of cost, morbidity, and suffering. Most people with depression are treated in general medicine using antidepressant medication. Outcomes are poor due to failure points across the care system, including patient non-adherence, failure of physicians to optimize the treatment regimens, and lack of patient-physician communication. This study reports on the 4week pilot deployment of MedLink, a mobile intervention aimed at systemically addressing each of these failure points. A mobile app provides the patient with information and collects data on symptoms and side-effects. A cellularly enabled pill bottle monitors medication adherence. Data from these are provided to the physician and patient to foster communication and medication adjustments. Usability evaluation was generally favorable. Medication adherence rates in this first deployment were high with no patients discontinuing, and $84 \%$ of doses taken. Depressive symptom severity was significantly reduced. This study supports the use of a comprehensive, systemic approach to mHealth solutions to enhance processes of care for depression by general medicine physicians.
\end{abstract}

Keywords—mental health; mHealth; adherence

\section{INTRODUCTION}

Major depressive disorder is common, affecting $7-10 \%$ in the general population in any given year and $20 \%$ of adults in their lifetime [1]. Depression imposes a very high societal burden in terms of cost, morbidity, suffering, and mortality [2], and is a leading cause of disability worldwide [3]. Very few people with depression ever see a mental health professional. The vast majority of people treated for depression are treated in general medicine with antidepressant medications.

Recovery rates among people treated for depression in general medicine are very low [4], due to a variety of factors related to the patient, the physician, and communication

This work was supported by research grant R34 MH095907 from the United States National Institutes of Health between the two. Patient adherence to antidepressants is low, with $60 \%$ of patients stopping their medications, usually in the first weeks or months $[5,6]$. This is substantially higher than the $30-50 \%$ non-adherence rates seen for non-psychiatric medications [7]. Even among those who continue taking their antidepressant medication, many do not take them every day as prescribed. General medicine physicians often do not manage medications according to treatment guidelines [8], and communication between patients and physicians is poor, aggravating the problem. Thus, a pervasive health solution to address problems in pharmacotherapy for depression in general internal medicine must address several areas systemically. We developed a mobile intervention, MedLink, to improve the quality of pharmacotherapy for depression in general medicine, based on a model of known person and system failure points, displayed in Figure 1.

There are a number of patient factors related to non-adherence. Patients frequently do not know how to take their medications, either because the prescribing physician did not adequately explain it, or because they do not remember. Patients do not know how to manage their symptoms of depression. Patients often do not know that they must take medications consistently for at least four weeks before they are likely to experience antidepressant benefits (although placebo benefits may occur earlier) [8]. Side effects, however, usually occur shortly after beginning treatment. While the severity of side effects frequently declines over time, patients are usually not provided with information on how to manage them. Patients usually receive no guidance on how to reduce forgetfulness (also a symptom of depression), such as taking the medication after another routine activity such as brushing teeth or having one's morning coffee. Many of the symptoms of depression for which the medication is prescribed, such as decreased motivation, hopelessness, and a sense that nothing will get better, paradoxically also contribute to non-adherence [9]. 


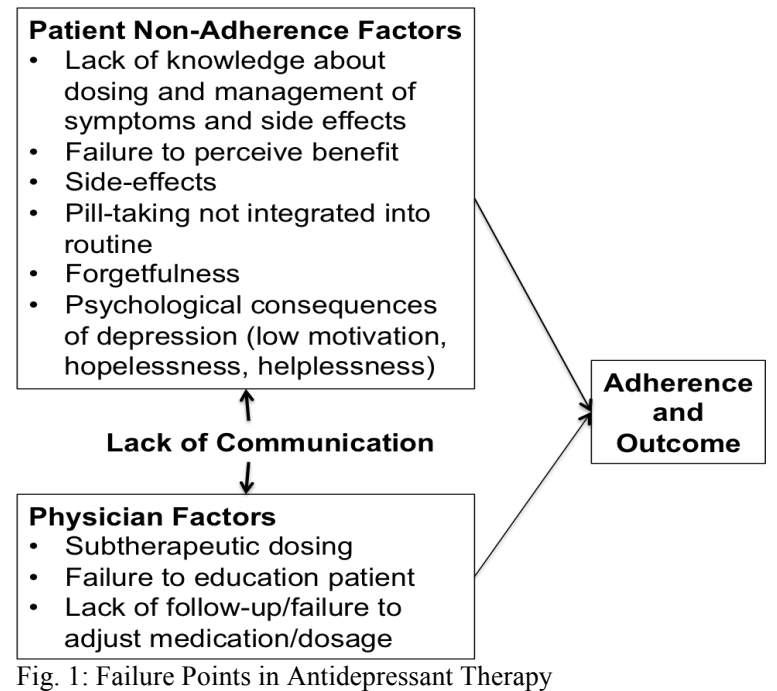

Thus, patients beginning antidepressant treatment for depression have little knowledge, initially experience side effects, expect but receive little immediate benefit, and are prone to forgetfulness, low motivation, hopelessness and helplessness, all of which increases the likelihood that they will discontinue their medications.

General practitioners typically do not follow-up with patients after a first prescription, as is recommended by treatment guidelines, which require follow-up every 4 weeks to optimize dose and treatment, and manage side effects [8]. Usually patients treated with antidepressants require multiple dose and/or medication changes to find a medication that minimizes side effects and a dose that is therapeutic. Effective doses often are 2-3 times the minimal clinical dosage at which treatment is usually initiated. Guideline concordant treatment does not fall into the normal workflow of most physicians, who typically prescribe medications that do not require ongoing adjustment and monitoring. Thus, the vast majority of primary care patients treated for depression are under-dosed [10]. Given the number of patient factors driving non-adherence, and the lack of communication between physicians and patients, and the failure of physicians to optimize the treatment regimen, it is not surprising that adherence rates are so low and treatment outcomes are so poor.

\section{A. Interventions for Adherence}

SMS reminders have been shown to improve adherence to many medications, although have not been investigated for antidepressants [11]. Devices that monitor pill-taking can also improve adherence if they are integrated into the process of care, such as providing adherence information to the care team [12]. However, these have also not been studied in the treatment of depression. Adherence to antidepressants can be improved by providing standardized patient education after the physician visit either through counseling or mailing information to the patient [13].

Even if patient adherence to antidepressants can be improved, this is unlikely to be effective in improving outcomes when medications and dosages are not optimized by treating physicians. Unfortunately, interventions aimed only at changing physician practice related to the management of depression, such as through decision support tools in EMR systems, do not to improve quality of care or outcomes [14]. Thus, a comprehensive strategy, focused on all potential failure points in the system, is required.

\section{MedLink Design}

MedLink is intended to support the care of patients initiating a new prescription for an antidepressant, as nonadherence usually occurs in the first weeks after a new prescription. MedLink is intended to support the establishment of durable medication taking behaviors, communication between the patient and physician, and decision making for the physician. MedLink has been designed to address each of the components in Figure 1. Communication with the physician was guided by our Supportive Accountability model [15], which indicates that patients are more likely to engage in behaviors if they know that a provider is receiving information about that behavior, and that they are likely to discuss that information at some point in the future. MedLink design was also informed by Wagner's Chronic Care Model, which has received considerable empirical support [16]. This model states that a care delivery system is most effective when 1) the patient is informed and activated to perform the prescribed tasks, 2) decision support tools are available to the care team to ensure guideline-concordant care, and 3) the care team is provided up-to-date information on the patient's status. Interventions that drive both the provider and patient to engage in care are more successful than those that address only the provider or patient alone. MedLink was designed to address each of the areas in Wagner's model, using existing knowledge about the patient and provider barriers to care.

MedLink was designed to include a patient-facing mobile app, a cellularly enabled pill bottle to track medication adherence, and physician decision support.

\section{A. Patient-Facing Elements}

The mobile app, developed for Android phones versions 4.1 or newer, provides information about medications, education about self-management skills, and collects symptom data. Screenshots are provided in Figure 2.

Patients are provided weekly "lessons" that include 5-10 screens of text containing evidence-based information on the management of depressive symptoms. The Week 1 lesson contains information on the management of antidepressant medications, tailored information regarding the patient's medication, dosage, and dose time (usually once per day in the morning), possible side-effects and side-effect management strategies, and expectations regarding medication effectiveness (including that it may take four or more weeks to notice a positive effect), and strategies for integrating medication taking into the patient's routine (such as doing it with an activity that is performed regularly, such as drinking morning coffee or brushing teeth). Subsequent weeks focus on common depression management strategies, such as increasing positive activities (Week 2), managing cognitive biases (Week 3) and improving social support (Week 4). The lessons are intended to address lack of knowledge about 
dosing and the time it takes for benefits to be perceived, management of side-effects, and addressing the psychological consequences of depression (low motivation, hopelessness, helplessness) by increasing personal agency through educating patients about basic self-management strategies for depression. We have found in previous studies that such information is highly valued by depressed patients $[17,18]$. Thus, the time-based release of information is intended to support continued engagement with MedLink.
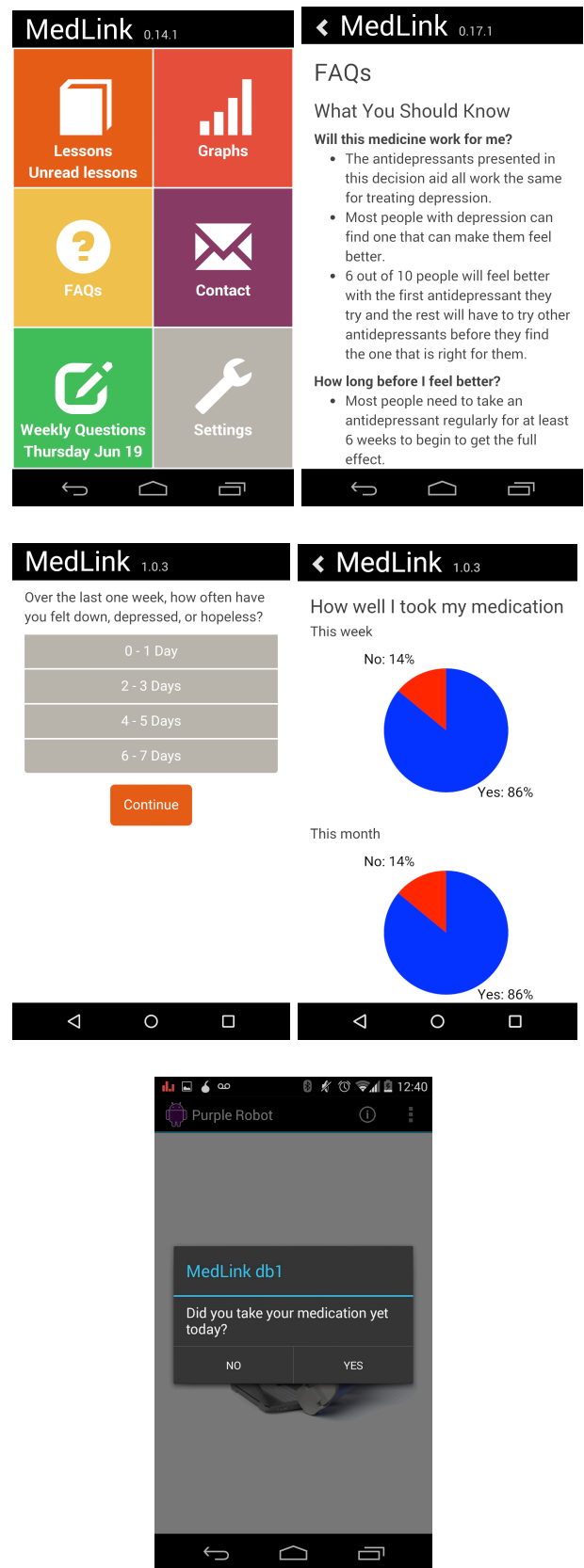

Fig. 2. MedLink Screenshots from top to bottom: Home Screen, Lesson, Assessment, Graphic Feedback, and Notification

Each week the patient is notified to complete in-app assessments of depressive symptom severity using the Patient
Health Questionaire-8 (PHQ-8) [19], a commonly used measure of depression, and the Frequency, Intensity and Burden of Side Effects (FIBSER) [20], to assess type and severity of side-effects. These assessments are released at the same time as the lesson to encourage their completion.

The app was intended to be used a minimum of 1-2 times per week to read the weekly lessons and complete questions.

Medication adherence is monitored using the Wisepill bottle, which emits a cellular signal when it is opened, allowing for near real-time acquisition of data regarding patient pilltaking activity. The app launches an in-phone reminder pop-up and audio alert 10 minutes after the scheduled dose time. At the same time, the pill bottle launches a visual alert (blinking light). To avoid notification fatigue, a cellular signal is sent to the phone when our servers detect a pill bottle opening, which prevents the launching of the reminder notifications on the phone. If MedLink does not receive notification that the pill bottle has been opened, the reminder is launched on the phone 10 minutes after the scheduled dose, asking if the patient has taken their medication. They are able to answer "yes," which allows us to accommodate system failures and obtain more accurate adherence data, or "no," which triggers another reminder 10 minutes later. The reminders are intended to address problems related to forgetfulness.

The MedLink app was subjected to in-lab usability testing with 23 patients taking medications (12 with major depression, and 11 without). These findings, reported elsewhere [21], identified minor problems, which were corrected. Patients indicated that they were uncomfortable with specific daily information on adherence being provided to their physician, such as a calendar representing on which days they were adherent or not adherent. However, they felt that providing overall adherence rates to physicians was acceptable. While patients reported that they would find an adherence support system such as MedLink useful, many were taking multiple medications for multiple medical problems and stated that they would prefer to manage all of their medications in a single system. While we find this reasonable, management of multiple medications is beyond the scope of this project. Overall, there were no differences between depressed and non-depressed patients in using the system, except in a task that involved interrupting the patient while engaging in a task in the app; depressed patients had significantly longer recovery times, however they were able to successfully recover.

\section{B. Reporting and Communication}

Every 4 weeks, the MedLink system generates a physician report (see Figure 3) that includes information on depressive symptom severity, side effects, adherence rates, and a treatment recommendation based on treatment guidelines. While these reports are intended to prompt the care team to contact the patient and to promote physician adherence to treatment guidelines, previous research has suggested that physicians are often not responsive to such decision support [14]. Therefore, as suggested by the Wagner Chronic Care Model, the same information is given to the patient. If a dose or medication change is recommended, the patient is provided the same information in the Physician Report and is prompted to contact 
the physician, and a contact phone number is provided. Thus, MedLink addresses lack of communication and lack of followup as failure points, by providing actionable information to both the care team and the patient, to increase the likelihood that communication and appropriate treatment management occurs.

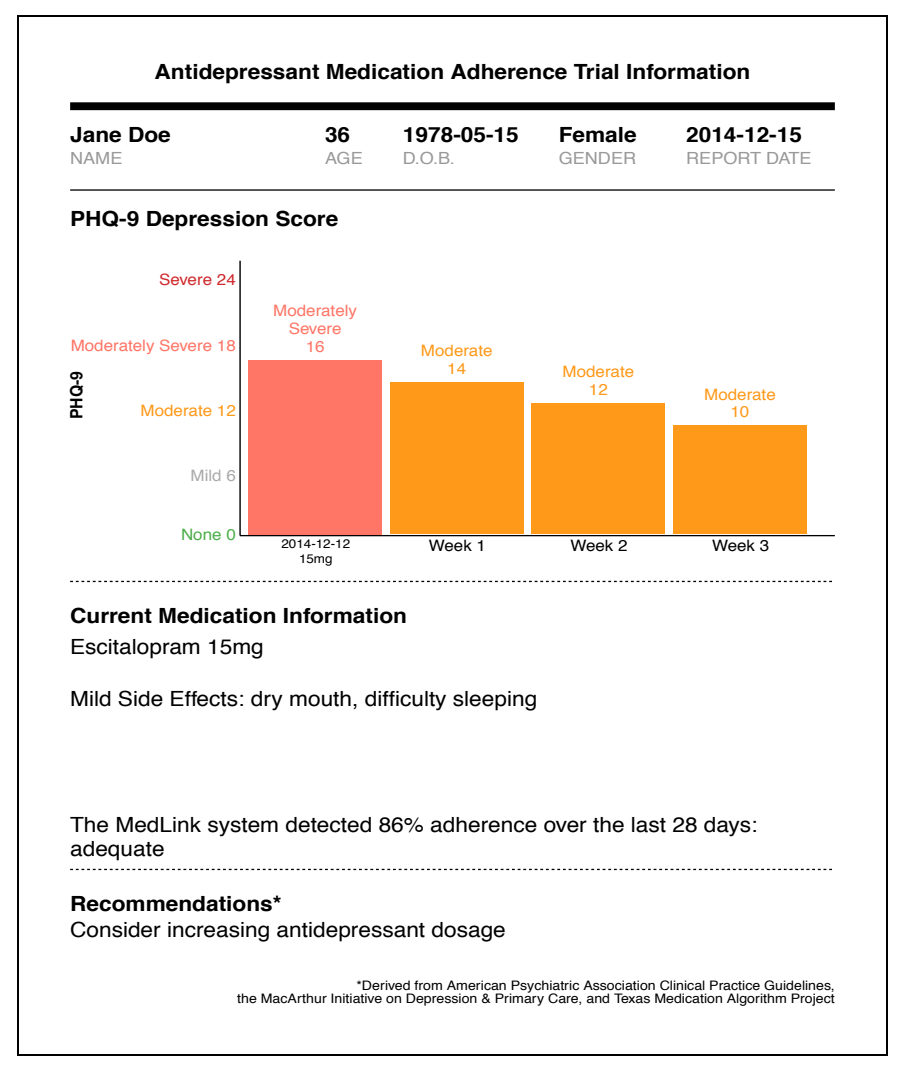

Fig. 3. Physician Report

\section{Physician-Facing Elements}

We conducted 2 focus groups with 10 physicians each. The first focus group explored the data elements that they would require, and how those data should be provided. Critical elements included information on depression severity, sideeffects, adherence, and decision support. Physicians were uniform in wanting weekly PHQ-8 scores, with an indicator of severity level (mild, moderate, severe) and a specific notification if there was an increase in score. The PHQ-8 is identical to the PHQ-9 [19], a standardized self-report tool commonly used in primary care to assess depression, but removes the item assessing suicidal ideation. The suicidal ideation item only assesses thoughts about death/suicide, which are common among depressed patients, and does not assess intent, and is therefore a poor indicator of risk. Physicians were concerned that the item assessing suicidal ideation is too non-specific to be actionable, and therefore would frequently trigger needless outreach to the patient, significantly increasing burden on the clinic staff and the patient. Information on the severity of side effects along with the specific side effect symptoms was also seen as important. Adherence rates as a percentage of days since the previous visit were requested as part of the report. There were some mixed feelings about specific treatment recommendations, with some stating it would be useful, and some expressing concerns that clear recommendations could present a legal liability if their clinical judgment led them to a course of action that was different from the recommendation. We elected to retain the treatment recommendation, given physician failure to optimize dose is a common failure point, and because treatment recommendations are a common part of current practice in American general medicine clinics.

There was no consensus on where such information could be provided in the electronic medical record (EMR) so that it would be noticed. Based on this information, we decided that for this early field trial we would provide paper reports to physicians. Three mock-ups were produced, with input from a general physician that included all requested elements. Information on guideline congruent treatment was softened to recommendations. These were presented to the group of physicians in the second focus group meeting, who uniformly preferred one mock-up and suggested minor modifications, which were made. The final Physician report is displayed in Figure 3.

\section{METHODS}

\section{A. Study Design}

The aim of the study was to evaluate MedLink in a representative group of patients to 1) ensure the system was functioning properly over time; 2) identify any problems in the system; 3) obtain user feedback; 4) evaluate adherence and depression severity. Adherence was defined in two ways: continuation vs. stopping the medication, and consistency (\% of days that the medication was taken). The evaluation period was 4 weeks, as this allowed us to evaluate all aspects of the system's functionality, including the provision of information on symptoms, side effects, and adherence to physicians and patients, as well as the occurrence of follow-up visits. In addition, the majority of patients who discontinue antidepressants do so within the first weeks of treatment.

Patients were recruited from Northwestern University's General Internal Medicine Clinic. When a physician made a new diagnosis of major depression and made a new prescription for an antidepressant, an alert for the study would pop up in the EMR. If patients indicated they were interested, they were contacted by study staff, who described the study and obtained informed consent for those willing to enroll. Patients who had acute suicidality, which included a suicide plan and the intent to carry out that plan, were excluded.

The MedLink app was installed on the patient's phone for those who had an Android device. Patients who did not have an Android phone were provided one with a full data and call plan.

Patients were asked to complete study surveys through the browser-based Redcap survey tool, completely outside the MedLink treatment system. Patients were informed that these data were for study purposes only, and would not be provided to their physicians, to avoid any reporting biases that might occur with in-app assessments that are intended for clinical 
communication. Depression outcomes were measured using the PHQ-9 through Redcap at baseline and week 4. Objective adherence was measured by the number of detected Wisepill bottle openings. Subjective adherence (consistency of pilltaking) was measured using the self-report Patient Adherence Questionnaire (PAQ) [22] at week 4. Usability for the entire MedLink system was measured at week 4 with the USE questionnaire [23]. To quantify the usability of each of the components, patients rated 4 statements drawn from the USE ("I like it," "It was easy to use," "I learned how to use it quickly," and "It was useful/helpful") for each component of MedLink (the educational lessons, weekly assessments, feedback graphs, and the Wisepill reminder system) using the 7-point likert scale, with 1 indicating strongly disagree and 7 indicating strongly agree. User feedback interviews were conducted to collect qualitative data regarding usability.

\section{RESULTS}

Eight patients judged by their physicians to meet criteria for major depression and prescribed an antidepressant were enrolled. Seven $(87.5 \%)$ patients were female, and they ranged in age from 22 to $62(\mathrm{M}=39.6)$. Three participants used their own phones, while 5 were provided study phones; of those 5 , one had an Android phone but no data plan, one had an Android phone that was too old, two had an iOS phone, and one had no smartphone. All patients completed the 4-week field trial.

\section{A. Medication Adherence and Depression Outcomes}

All patients were continuing to take their medications at week 4. Consistency of adherence, monitored using the pillbox, indicated that $72.5-100 \%$ of doses were taken $(\mathrm{M}=$ $84 \%$ ). Based on the week 4 PAQ self-report, 5 (62.5\%) patients reported taking their antidepressant medication every day, 2 (25\%) reported missing a dose approximately once per week, and 1 reported missing doses more frequently due to side effects. While it is possible that our sample was biased towards people who were more likely to adhere to treatment, these findings are nonetheless encouraging given that approximately $40 \%$ of patients treated with antidepressants in general medicine would be expected to be continuing to take their antidepressant during this period $[5,6]$.

Depression outcomes on the PHQ-9 assessment administered for research purposes were analyzed using a Wilcoxon signed rank test. Patients were moderately depressed at baseline, with the median PHQ-9=12.5 (interquartile range $=9-16$ ), dropping after 4 weeks to mild (median PHQ-9=5, interquartile range $=2.5-10 \% ; p=0.008$ ). This decline in the severity of depressive symptoms was mirrored by the in-app PHQ-8 data being provided to physicians, with a median score at baseline of 12 (interquartile range $=9-14.5 \%$ ), dropping over the 4 weeks to 5 (interquartile range $=2.5-10 \% ; p=0.008)$. Thus, the PHQ depression assessments administered provided to physicians was nearly identical to the browser-based PHQ administered for research outcomes, suggesting little or no bias in symptom reporting to physicians. It should be noted that patients were enrolled in the study on average $17 \quad(\mathrm{SD}=3.2)$ days after receiving their prescription, due to time required to contact, assess, consent, and enroll them. Thus, the study baseline likely does not represent patients' symptom severity levels at the start of treatment. Nevertheless, these outcomes suggest that patients, who began the study with moderate levels of symptoms in the range where treatment is recommended (guidelines recommend treatment for those with a PHQ-9 $\geq 10$ [24]), fell at the low end of mild symptoms by the end of treatment. In clinical application, the MedLink system would best be provided to patients in a clinic or pharmacy to reduce the lag time between initiation of medications and monitoring.

\section{B. Usability Outcomes}

Patients launched the MedLink app an average of 17.4 $(\mathrm{SD}=8.5)$ times over the course of the 4 weeks. The average ratings on the USE questionnaire were $4.8(\mathrm{SD}=0.8)$ for Satisfaction, $5.7(\mathrm{SD}=1.1)$ for Ease of Use, $6.1(\mathrm{SD}=1.5)$ for Learnability, and $4.6(\mathrm{SD}=1.0)$ for Usefulness. While these are all generally positive, likeability and usefulness were closer to the neutral point. Satisfaction was pulled down by some items, such as "it was fun to use," $(\mathrm{M}=3.75)$ which was not the intent of the application. Other items addressing satisfaction $(\mathrm{M}=6.0)$ or inclination to recommend it $(M=5.75)$ were high. Similarly, for Usefulness, items that were less relevant to MedLink, such as "It helps me be productive" $(\mathrm{M}=4.25)$ and "It gives me more control over my activities" $(M=3.0)$ were low, while "It is useful" (M=5.5) and "It does everything I would expect it to" $(M=5.5)$ were higher. More detailed usability evaluation was conducted through ratings and interviews for each of the components of the application.

\section{1) Lessons}

Seven of the 8 patients read the lessons for week 1 (Welcome to MedLink; general information on medication adherence) and week 2 (Getting Activated!; information on increasing positive activities), 4 read the week 3 lesson (Thoughts; information on managing depressive cognitive biases), and 5 read the week 4 lesson (Increasing Social Activity; strategies to improve social support). One user indicated that she was unaware of the lessons and did not notice the change in the tile on the home screen.

Usability among those who viewed the lessons was generally rated as positive, averaging $6.0(\mathrm{SD}=1.1)$ for liking, $6.6(\mathrm{SD}=0.5)$ for ease of use, $6.6(\mathrm{SD}=0.5)$ for learnability, and $5.8(\mathrm{SD}=1.7)$ for usefulness. In user feedback interviews several people mentioned spontaneously that the lessons were their favorite part of the MedLink app. For example a representative comment was "I like the lessons... They're full of helpful advice or offer perspective that I really wouldn't have thought about with handling depression."

While prompts for the lessons were provided through tray notifications, 2 users (including the one who did not view the lessons) indicated that they did not use the tray and would have liked email or text reminders when the lessons became available. One user commented that she stopped viewing the lessons because she was in therapy and the information wasn't new. Two of the 8 participants were in concurrent psychotherapy. These findings suggest that while the lesson content was not accessed uniformly, it is a valuable component 
of the intervention, except when the patient is in psychotherapy.

\section{2) In App Assessments}

Of the 32 assessments, 29 (96.6\%) were completed. Usability for the in-app depression and side effect assessments was generally rated as positive, averaging $5.1(\mathrm{SD}=1.1)$ for liking, $5.5(\mathrm{SD}=1.2)$ for ease of use, $6.1 \quad(\mathrm{SD}=1.4)$ for learnability, and $6.1(\mathrm{SD}=1.4)$ for usefulness, indicating overall satisfaction. Feedback interviews revealed that patients were generally neutral about the assessments. This is consistent with general experience in clinical research, where participants are incentivized through payments to complete self-report assessments. The high rate of completion, in the absence of incentivization, appeared in part due to their awareness that this information was being provided back to their physician. This is consistent with our theory of supportive accountability [15], which argues that patients are more compliant with tasks when they know that their care provider can see their activity. The feedback graphs from the assessments generated a range of responses. In contrast to the positive ratings, 3 of the patients reported on interview that they were not very useful ("They didn't provide any new information"). The other half liked them. One stated "It's nice to see it graphically; I'm a kind of a scientist, and very visual." Another reported showing them to her therapist each week.

\section{3) Pill Bottle and Reminder Prompts}

Reminders were launched on $76(30.4 \%)$ of 250 study days. Participants indicated for $58(76.3 \%)$ of these reminders that they had already taken their medication. While the bottle is cellularly enabled, the median time for our servers to receive the notification was 32 minutes ( $\mathrm{IQR}=8-112 \mathrm{~min}$ ), with 126 $(68 \%)$ arriving more than 10 minutes after their scheduled medication dose time. Thus, reminders frequently arrived after patients had already taken their medications, due to connectivity problems with the Wisepill bottle. While delays occurred to some extent for all patients, they were more pronounced for some patients, likely due to poor connectivity in their homes. Reminders were also more likely to be launched on weekends, likely due to changes in people's schedules affecting when they took the medications.

The appearance of reminders after taking the medication was a common source of confusion among patients, who commented during user feedback interviews that "I received prompts even after opened my pill box. I didn't know if the pillbox was registering that it had been opened." Another user complained that the medication reminder pop-up interrupted her activity on the phone. There was also some confusion about how the prompts worked. For example, one user commented, that the reminders should include an audio reminder. The prompt in fact does include an audio alert, however, this requires that the phone have sounds turned on.

In spite of these problems, the use of a cellularly enabled pill bottle to provide reminders was rated highly, with means scores of $5.4(\mathrm{SD}=0.7)$ for liking, $6.4(\mathrm{SD}=0.7)$ for ease of use, $6.4(\mathrm{SD}=0.7)$ for learnability, and $6.0(\mathrm{SD}=1.4)$ for usefulness. Evidence that MedLink prompts, in spite of the problems in preventing their launching, were promoting habit formation around medication adherence was evidenced for one user who commented that she "required fewer reminders by end of the study, as taking my medication has become a habit."

One patient reported having difficulty getting the pill bottle through airport security. While we did not anticipate this particular problem, it is indicative of the wider systemic issues that may be present for such adherence systems. Other systemic issues that we considered were related to privacy concerns, such as receiving reminders in public.

\section{4) Follow-up appointments}

Seven of the 8 patients had follow-up physician contact to monitor and adjust medications during the trial or shortly after. There were 10 contacts in total, including 6 office visits, 4 by telephone, and 2 through the patient messaging portal in the EMR system. Given that follow-up visits and dose adjustments rarely occur in general internal medicine, these findings suggest that MedLink was effective at improving communication and processes of care for these patients.

\section{IMPLICATIONS FOR DESIGN}

MedLink is a mobile system designed specifically to address a comprehensive set of failure points in the treatment of depression in general internal medicine, involving patient adherence, physician management of the medication regimen, and reporting to prompt patient-physician communication. We review here some of the specific lessons learned, as well as a broader set of issues raised by this work.

Regarding the patient facing features, patient notifications require some adjustments. While initial in lab usability did not uncover the problems with tray notifications to let users know when new lessons or content was release, this emerged as a problem or complaint in the field test. Given people use different methods to receive such information, allowing greater flexibility for the user to select the notification method would be important. However, MedLink did have a Settings section where users could make minor adjustments to the app, such as font size. This feature was accessed by only one user, suggesting that while offering user control through Settings might be worth including, it would likely not solve the problem. Forcing a choice during app setup is another commonly used option. However, this asks users to make choices about interaction methods before they have experience with the app and thus may also result in suboptimal selections. Both of these will be implemented in the next version, however, ultimately in-phone analytics may be useful that can suggest refinements to the notification methods as patterns of response and non-response are detected.

The medication reminders, while valued, presented a number of problems. Our inability to prevent many of the reminders when the medication had already been taken contributed to some confusion as to their purpose, likely because the reminders were not perceived as connected to a specific context (i.e., not taking a medication). While such connectivity problems may improve over time, in the more immediate time frame, they are beyond our control. However, within the current connectivity limitations, some solutions may mitigate the problem. Extending the amount of time between 
the expected dosing time and the reminder would reduce the number of unneeded reminders. For example, rather than define the reminder time in reference to the usual time the person takes their medication, we could base the alert on the latest time they could usually receive a reminder and still be able to take the medication. Allowing the timing of the alert to vary based on work and non-work days might also reduce the number of unneeded reminders. Finally, making the alert rules transparent to the user, for example stating, "MedLink is reminding you to take your 9am dose of [medication]" may reduce confusion when the reminders are launched in error.

Lessons appeared to be valued components of the intervention, underscoring patients' desire for information about both their medications and the management of depression, at least for those not currently in psychotherapy. Patients typically view their problem as having depression and not as having a problem with medication adherence. Providing support that meets their goals of feeling better likely offers a rationale for the use of the system that is more consistent with their goals. Providing this information over time may also support more sustained engagement by continuing to provide content that is of value.

Patients completed a very high percentage of the in-app self-report symptom assessments. While the elevated responding likely was due in part to the patient's knowledge that these results were being provided to their physicians, we suspect it is an overestimation of what assessment completion would be in clinical deployment, as these were research subjects who had agreed to complete research assessments. Thus, the functionality and usefulness of the system with missing information (e.g., missing data on depressive symptoms and side effects) in the physician reports will have to be evaluated in larger deployments.

Physicians were very clear in the focus groups that anything that increased workload or did not fit into their usual workflow would likely not be accepted. Integrating information into the EMR will be a critical step in making MedLink scalable. The problem of where and how to present these data within the EMR is complicated by the fact that physicians use EMRs differently, and there are many different EMR systems. As EMRs begin developing hooks for mobile data, these problems will become common, and this is an area of ongoing research.

More broadly, unlike many commercially available medication adherence systems, which are largely agnostic to the clinical problem or medication, MedLink takes a disease specific approach. This presents both opportunities and challenges. Challenges to pharmacotherapy can be divided into those that are common across many diseases and medications (e.g., forgetfulness, formation of pill-taking habits), and those that are specific to a disease or class of medications.

A disease specific approach opens the opportunity to address a comprehensive set of failure points in pharmacotherapy that include specific patient issues (e.g., specific side-effects, specific challenges in the population such as motivation, or understanding of the treatment regiment) and medication management issues. In addition, a disease specific approach allows the system to integrate disease management strategies into pharmacotherapy management, thereby aligning the goals of the system more closely with the patient's goals of wellness.

However, a common concern raised in initial lab usability testing was that most patients are taking multiple medications for multiple problems, and want to have a single system for managing all of their medications [21]. This paradox opens a number of challenges. First, a system that provides disease specific support for multiple common chronic problems encountered in general medicine (depression, hypertension, diabetes, etc.), would have to include specific information on a wide variety of medications and side effects, and diseasespecific self-management strategies. This would require developing methods of providing diverse types of information and prompts to people in a way that does not confuse or overwhelm them.

A second set of problems related to managing multiple medications concerns the hardware for monitoring medications, which often are taken at different frequencies and times of the day. Using individual pill bottles presents safety issues; if patients place medications in the wrong bottle, adherence data will be incorrect and erroneous reminders will be launched. Trays that require presorting of medications are often rejected by patients as too much trouble and increase the possibility of errors. Some solutions are available that batch and package medications either at the pharmacy or in home, however it is difficult to manage medication and dosing changes that can occur frequently. Thus, the development of hardware solutions for the management of medications remains an ongoing area of research and development.

Comprehensive, disease specific methods of improving pharmacotherapy adherence and outcomes has many potential benefits, and are likely to be more effective for the specific disease and medication. But people frequently do not have only one medical problem. Currently we are confronted with a situation where increasing the specificity of the solution comes at the cost of generalizability across the multiple medical problems and medications that are part of the care for many general medicine patients. Narrowing the divide between specificity and generalizability will be an important focus of research in mobile solutions to improve pharmacotherapies in general medicine.

Solutions that focus only on depression management have frequently addressed just one or a subset of failure points such patient forgetfulness [12], lack of patient knowledge [13], or physician actions [14]. Addressing adherence as a systemic problem appears to have value. Adherence to the depression assessment was remarkably high, in part because patients were aware that their information was being provided to their physician. Similarly, the high medication adherence rates were also likely due in part to patients' awareness that adherence information was being transmitted to their physicians. This bolsters supportive accountability theory [15], which states that patients are more likely to perform expected behaviors if they know that their physician is receiving information about their completion. 
The present study has a number of limitations. As this was an initial field trial, it was only intended to test the functionality of the system over time. While the adherence rates far exceeded what would be expected in general clinical practice, it is possible, and indeed likely, that the patient sample was not representative of the average clinic patients starting an antidepressant medication. In addition, dose and medication adjustments should continue for several months, until remission of depression has been achieved. We are currently beginning a 12 -week randomized controlled trial to evaluate whether MedLink improves medication adherence and depression relative to usual care, which will answer some of these questions.

Finally, the current study cannot tell us if any of the components of the intervention are particularly effective, if some are useful, or if each component is serving the specific intended function, such as increasing knowledge or motivation. Such questions can be answered conclusively with factorial trials that randomly provide specific components.

\section{CONCLUSION}

The limitations not withstanding, the present study provides initial evidence for MedLink. MedLink approaches pervasive health from a systems approach, addressing a comprehensive set of known failure points in processes of pharmacotherapy for depression in general medicine. These include improving patient knowledge and motivation, preventing forgetfulness, reporting and treatment recommendations to foster patientphysician communication, and prompting physicians and patients to ensure guideline concordant care, thereby improving medication adherence and depression outcomes.

\section{REFERENCES}

[1] R. C. Kessler, P. Berglund, O. Demler, R. Jin, D. Koretz, K. R. Merikangas, et al., "The Epidemiology of Major Depressive Disorder: Results From the National Comorbidity Survey Replication (NCS-R)," Jama, vol. 289, pp. 3095-105, Jun 182003.

[2] K. B. Wells, A. Stewart, R. D. Hays, M. A. Burnam, W. Rogers, M. Daniels, et al., "The functioning and well-being of depressed patients. Results from the Medical Outcomes Study," Jama, vol. 262, pp. 914-9, Aug 181989.

[3] A. J. Ferrari, F. J. Charlson, R. E. Norman, S. B. Patten, G. Freedman, C. J. Murray, et al., "Burden of depressive disorders by country, sex, age, and year: findings from the global burden of disease study 2010," PLoS Med, vol. 10, p. e1001547, Nov 2013.

[4] D. A. Regier, W. E. Narrow, D. S. Rae, R. W. Manderscheid, B. Z. Locke, and F. K. Goodwin, "The de facto US mental and addictive disorders service system. Epidemiologic catchment area prospective 1year prevalence rates of disorders and services," Arch Gen Psychiatry, vol. 50, pp. 85-94, Feb 1993.

[5] M. Olfson, S. C. Marcus, M. Tedeschi, and G. J. Wan, "Continuity of antidepressant treatment for adults with depression in the United States," Am J Psychiatry, vol. 163, pp. 101-8, Jan 2006.

[6] V. M. Hunot, R. Horne, M. N. Leese, and R. C. Churchill, "A Cohort Study of Adherence to Antidepressants in Primary Care: The Influence of Antidepressant Concerns and Treatment Preferences," Prim Care Companion J Clin Psychiatry, vol. 9, pp. 91-99, 2007.
[7] E. Vermeire, H. Hearnshaw, P. Van Royen, and J. Denekens, "Patient adherence to treatment: three decades of research. A comprehensive review," J Clin Pharm Ther, vol. 26, pp. 331-42, Oct 2001.

[8] American Psychiatric Association, "Practice guideline for the treatment of patients with major depressive disorder (revision)." Am J Psychiatry, vol. 157, pp. 1-45, Apr 2000.

[9] M. Pompili, G. Serafini, A. Del Casale, S. Rigucci, M. Innamorati, P. Girardi, et al., "Improving adherence in mood disorders: the struggle against relapse, recurrence and suicide risk," Expert Rev Neurother, vol. 9, pp. 985-1004, Jul 2009.

[10] G. E. Simon, E. H. Lin, W. Katon, K. Saunders, M. VonKorff, E. Walker, et al., "Outcomes of "inadequate" antidepressant treatment," $J$ Gen Intern Med, vol. 10, pp. 663-70, Dec 1995.

[11] M. Vervloet, L. van Dijk, J. Santen-Reestman, B. van Vlijmen, P. van Wingerden, M. L. Bouvy, et al., "SMS reminders improve adherence to oral medication in type 2 diabetes patients who are real time electronically monitored," Int J Med Inform, vol. 81, pp. 594-604, Sep 2012.

[12] K. D. Checchi, K. F. Huybrechts, J. Avorn, and A. S. Kesselheim, "Electronic medication packaging devices and medication adherence: a systematic review," JAMA, vol. 312, pp. 1237-47, Sep 242014.

[13] L. Hoffman, J. Enders, J. Luo, R. Segal, J. Pippins, and C. Kimberlin, "Impact of an antidepressant management program on medication adherence," Am J Manag Care, vol. 9, pp. 70-80, Jan 2003.

[14] B. L. Rollman, B. H. Hanusa, H. J. Lowe, T. Gilbert, W. N. Kapoor, and H. C. Schulberg, "A randomized trial using computerized decision support to improve treatment of major depression in primary care," $J$ Gen Intern Med, vol. 17, pp. 493-503, Jul 2002.

[15] D. C. Mohr, P. Cuijpers, and K. Lehman, "Supportive Accountability: A Model for Providing Human Support to Enhance Adherence to eHealth Interventions," J Med Internet Res, vol. 13, p. e30, 2011.

[16] E. H. Wagner, "Chronic disease management: what will it take to improve care for chronic illness?," Eff Clin Pract, vol. 1, pp. 2-4, AugSep 1998.

[17] D. C. Mohr, J. Duffecy, L. Jin, E. J. Ludman, A. Lewis, M. Begale, et al., "Multimodal e-mental health treatment for depression: a feasibility trial," J Med Internet Res, vol. 12, p. e48, 2010.

[18] D. C. Mohr, J. Duffecy, J. Ho, M. Kwasny, X. Cai, M. N. Burns, et al., "A Randomized Controlled Trial Evaluating a Manualized TeleCoaching Protocol for Improving Adherence to a Web-Based Intervention for the Treatment of Depression," PLoS One, vol. 8, p. e70086, 2013.

[19] K. Kroenke, R. L. Spitzer, and J. B. Williams, "The PHQ-9: validity of a brief depression severity measure," J Gen Intern Med, vol. 16, pp. 60613, Sep 2001.

[20] S. R. Wisniewski, A. J. Rush, G. K. Balasubramani, M. H. Trivedi, and A. A. Nierenberg, "Self-rated global measure of the frequency, intensity, and burden of side effects," J Psychiatr Pract, vol. 12, pp. 71-9, Mar 2006.

[21] E. Montague, C. Stiles-Shields, and D. C. Mohr, "Usability evaluation of MedLink to improve pharmacotherapy in primary care," in 58th International Meeting of the Human Factors and Ergonomics Society, Chicago, IL, 2014.

[22] M. A. Chesney, J. R. Ickovics, D. B. Chambers, A. L. Gifford, J. Neidig, B. Zwickl, et al., "Self-reported adherence to antiretroviral medications among participants in HIV clinical trials: the AACTG adherence instruments. Patient Care Committee \& Adherence Working Group of the Outcomes Committee of the Adult AIDS Clinical Trials Group (AACTG)," AIDS Care, vol. 12, pp. 255-66, Jun 2000.

[23] A. M. Lund, "Measuring usability with the USE Questionnaire," STC Usability Newsletter, vol. 8, 2001.

[24] The MacArthur Foundation's Initiative on Depression and Primary Care, "The Macarthur initiative on depression and primary care at Darmouth and Duke: Depression management toolkit," Dartmouth, Hanover, NH2004. 